\title{
Acclimatization in wide dynamic range multichannel compression and linear amplification hearing aids
}

\author{
E. William Yund, PhD; ${ }^{1 *}$ Christina M. Roup, PhD; ${ }^{1}$ Helen J. Simon, PhD; ${ }^{2}$ Glen A. Bowman, BA ${ }^{\mathbf{1}}$ \\ ${ }^{1}$ Sensorineural Hearing Loss Research Laboratory, Department of Veterans Affairs Northern California Health Care \\ System, Martinez, CA; ${ }^{2}$ The Smith-Kettlewell Eye Research Institute, San Francisco, CA
}

\begin{abstract}
Acclimatization was studied in hearing-impaired patients with no previous hearing aid (HA) experience who were fit bilaterally with either wide dynamic range multichannel compression (WDRMCC) or linear amplification (LA) HAs. Throughout 40 weeks of normal HA use, we monitored changes in nonsense syllable perception in speech-spectrum noise. Syllable recognition for WDRMCC users improved by $4.6 \%$ over the first 8 weeks, but the $2.2 \%$ improvement for LA users was complete in 2 to 4 weeks. Consonant confusion analyses indicated that WDRMCC experience facilitated consonant identification, while LA users primarily changed their response biases. Furthermore, WDRMCC users showed greater improvement for aided than unaided stimuli, while LA users did not. These results demonstrate acclimatization in new users of WDRMCC HAs but not in new users of LA HAs. A switch in amplification type after 32 weeks produced minimal performance change. Thus, acclimatization depended on the type of amplification and the previous amplification experience.
\end{abstract}

Key words: acclimatization, hearing aid, hearing aid experience, hearing impairment, hearing loss, linear amplification, rehabilitation, speech cues, speech perception, syllable recognition, wide dynamic range multichannel compression.

\section{INTRODUCTION}

Acclimatization refers to improvements in speech perception that occur as an individual learns to use the speech cues provided by a new hearing aid (HA) [1]. A primary reason for interest in acclimatization is that it represents improvements in speech perception that are not immediately available to the new HA user. Such unrealized benefits can have broad implications: the patient must wait to receive the full benefit of a new HA. The audiologist may not be able to use the patient's initial reactions to the HA as a guide toward optimal settings for future performance. Researchers cannot measure the optimal performance for any HA without allowing time for acclimatization to be completed. Furthermore, if acclimatization takes a long time or stops before it is complete, developed methods for facilitating acclimatization would be beneficial so that patients could reliably

\footnotetext{
Abbreviations: $\mathrm{ANOVA}=$ analysis of variance, $\mathrm{CV}=$ consonantvowel, $\mathrm{FFT}=$ fast Fourier transformation, $\mathrm{HA}=$ hearing aid, HAPI = Hearing Aid Performance Inventory (questionnaire), $\mathrm{HL}=$ hearing level, ITC = in-the-canal, LA = linear amplification, MMSE = Mini-Mental State Examination, NAL-R = National Acoustic Laboratory-Revised, NCHCS = Northern California Health Care System, NST = nonsense syllable test, $\mathrm{PC}$ = personal computer, $\mathrm{PHAB}=$ Profile of Hearing Aid Benefit (questionnaire), SNR = signal-to-noise ratio, SPL = sound pressure level, VA = Department of Veterans Affairs, VC = vowel-consonant, WDRMCC = wide dynamic range multichannel compression.

* Address all correspondence to E. William Yund, PhD; VA Research Service, Northern California Health Care System/151, 150 Muir Rd, Martinez, CA 94335; 925-372-2296; fax: 925-228-5738. Email: yund@ebire.org

DOI: 10.1682/JRRD.2005.06.0099
} 
obtain the maximum benefit in the minimum amount of time.

Questions about the speed, magnitude, and practical importance of acclimatization remain controversial. Some studies suggest that acclimatization is very rapid, or perhaps does not exist at all, because speech perception measured immediately after fitting (or within a day or two) was as good as that measured after months of HA experience [2-12]. Others have found continuing improvement in speech perception after 10 to 18 weeks of experience with new HAs [13-21].

Considerable variation across acclimatization results may not be surprising because of the many factors that have differed across studies [1,22-23]. Methodological differences among studies include: (1) subject factors such as age, hearing impairment, and previous HA experience; (2) HA factors such as type and number of HAs and the prescriptive formula for fitting; and (3) measurement factors such as the questionnaires and/or speech perception tests, the time schedule of the measurements, and the reference (or control) conditions against which performance changes were evaluated.

Many conditions are critical if a positive finding of acclimatization is to be obtained:

1. The auditory experience outside the laboratory with the new HAs must be sufficiently different from the user's previous experience that something can be learned. Since these changes in auditory experience accompany the use of new HAs, we refer to these changes collectively as "new information." The type of new information should determine the expected nature of the acclimatization. The new HAs, the old HA(s) or unaided hearing, and the auditory environment will all affect acclimatization.

2. If enough new information exists, then a learning process can occur. Whether learning must be triggered by discrete events or whether speech perception continuously adapts for maintenance of good performance, a shift toward the use of the new information is the essence of acclimatization. In either case, the path to significant improvement could include one or more performance decrements that might delay or halt progress toward optimal acclimatization to the new HA. Individual differences in sensitivity to environmental change and in learning ability as well as the duration and type of HA use will affect this condition.

3. Tests must be sensitive to the acclimatization that has occurred. Although a broad range of knowledge and skills are required for normal speech perception, tests emphasizing semantic knowledge or contextual inference would not be optimally sensitive to acclimatization, which would occur primarily on the level of phoneme identification. Similarly, the test material should require the use of auditory information that was compromised by the hearing impairment and at least partially restored by the new HA. Acclimatization to auditory information that was unaffected by the hearing impairment or the new HA should not be measured.

Hearing impairment affects speech perception in more complex ways than merely reducing audibility. Hearing-impaired and normal-hearing adults show differential use of speech recognition cues [24-33]. In general, hearing-impaired listeners relied less on high-frequency cues preferred by normal-hearing listeners, when hearing loss compromised those cues. Instead, they relied more on cues that were not disturbed by the hearing loss. Although greater reliance on secondary speech cues might reduce speech-recognition accuracy if primary cues were equally available, accuracy would increase after the primary cues had become unreliable because of hearing loss. Thus, during the acquisition of their sloping high-frequency hearing loss, subjects like those in the present experiment should have shifted away from reliance on high-frequency speech cues. When such subjects get HAs that increase access to high-frequency cues, acclimatization implies, by definition [1], increased use of high-frequency speech cues and improved recognition of high-frequency phonemes, like plosive and fricative consonants.

Our previous results indicate that wide dynamic range multichannel compression (WDRMCC) signal processing effectively provided consistent high-frequency speech cues as signal-to-noise ratio (SNR) decreased or as speech intensity decreased in constant noise [34-35]. This consistency may facilitate acclimatization, and we have reported a strong acclimatization-like effect in experiments in which subjects listened to WDRMCC stimuli in extensive laboratory test sessions ( $>150 \mathrm{~h}$ ) but had no WDRMCC experience outside of the laboratory [36]. The two HAs in the present experiment have WDRMCC signal processing similar to the HAs in our previous experiments and would be expected to provide similarly consistent high-frequency speech cues.

High-frequency speech cues are particularly important for speech recognition in speech-spectrum noise because the noise effectively masks low-frequency 
speech cues, with less masking at higher frequencies. An individual with a sloping high-frequency hearing loss has difficulty hearing speech in speech-spectrum noise because of the combined loss of high-frequency cues from the impairment and low-frequency cues from masking by the noise. When normal-hearing individuals are confronted with speech in speech-spectrum noise, the redundancy of speech permits them to rely on predominantly high-frequency information. We can use these stimulus conditions to determine whether the option to rely on high-frequency speech cues becomes available again to the new HA user as a result of acclimatization.

The present experiment consisted of two phases for two groups of new HA users. In the first phase, one group used HAs programmed for WDRMCC signal processing and the other used HAs programmed for linear amplification (LA). We measured the acclimatization effects of normal use of these HAs in sequential nonsense syllable tests (NSTs) in the laboratory. Initially, none of these subjects had any previous experience with HAs, but after phase 1, each had 32 weeks of experience with either WDRMCC or LA HAs. At the beginning of phase 2, the signal processing in all subjects' HAs was switched, either from WDRMCC to LA or LA to WDRMCC, and acclimatization was measured for 8 additional weeks. As a result, acclimatization was measured under four different conditions: (1) WDRMCC use with no previous experience, (2) LA use with no previous experience, (3) WDRMCC use with previous LA experience, and (4) LA use with previous WDRMCC experience. These two types of HA signal processing are quite different: WDRMCC has fast acting, independent compression over a large intensity range in a large number of channels, while in contrast, LA has no nonlinearity below the level of output limiting. If no clear differences were noted between the initial acclimatization to WDRMCC and LA, it would indicate that acclimatization in new HA users does not depend strongly on the type of HA amplification. If clear acclimatization effects were not found when HA users switched from LA to WDRMCC or WDRMCC to LA, it would indicate that relatively little acclimatization should be expected for a switch between two HAs, even if their signal-processing algorithms are quite different. The implications of clear acclimatization differences between LA and WDRMCC HAs will be a primary topic for discussion.

Although the present study consists of a single large experiment, it will provide answers to a number of spe- cific questions: (1) Will normal HA use produce increased syllable recognition on the NST? (2) What is the time course of this performance change? (3) Will results differ for users of different types of HA signal processing? (4) Will changes in consonant confusions differ for different types of HA signal processing? (5) Will the initial users of one type of signal processing show further acclimatization after they switch to the other type? (6) Will the Profile of Hearing Aid Benefit (PHAB) and Hearing Aid Performance Inventory (HAPI) questionnaires indicate acclimatization similar to that seen with the NST?

\section{METHODS}

\section{Subjects}

The 39 individuals who participated in this study were recruited from the Department of Veterans Affairs (VA) Northern California Health Care System (NCHCS) Audiology Service (Martinez, California) and from the surrounding community. The group included 29 men and 10 women who ranged in age from 43 to 84 years (mean age \pm standard deviation $=66.7 \pm 9.3$ ). No one in the group had used HAs before. Informed consent was obtained from all subjects in accordance with the standards of the VA institutional review board.

To ensure that hearing impairments were as homogenous as possible across subject groups, we selected individuals by audiometric configuration and apparent etiology consistent with presbycusis and/or noise exposure, with gradual onset after puberty. All subjects had sloping bilaterally symmetrical (within $15 \mathrm{~dB}$ ) audiometric configurations, with a threshold $45 \mathrm{~dB}$ hearing level (HL) at $500 \mathrm{~Hz}$ that gradually sloped to $75 \mathrm{~dB} \mathrm{HL}$ at $4 \mathrm{kHz}$. Thresholds were always at least $20 \mathrm{~dB}$ greater at $4 \mathrm{kHz}$ than at $500 \mathrm{~Hz}$, and threshold changes were no greater than $40 \mathrm{~dB}$ in one octave. Figure 1 shows the average audiometric thresholds for the subjects according to their first HA experience (LA or WDRMCC) [37]. All subjects were in good physical health with normal daily functioning and had no history of unusual noise exposure, chronic disease, alcoholism, drug abuse, neurological disorders, or severe hypertension. No subjects were receiving treatment with psychopharmaceutical agents or ototoxic drugs. Cognitive abilities were verified by a Mini-Mental State Examination (MMSE) score of at least 25 [38]. Because of the extended duration and relative 


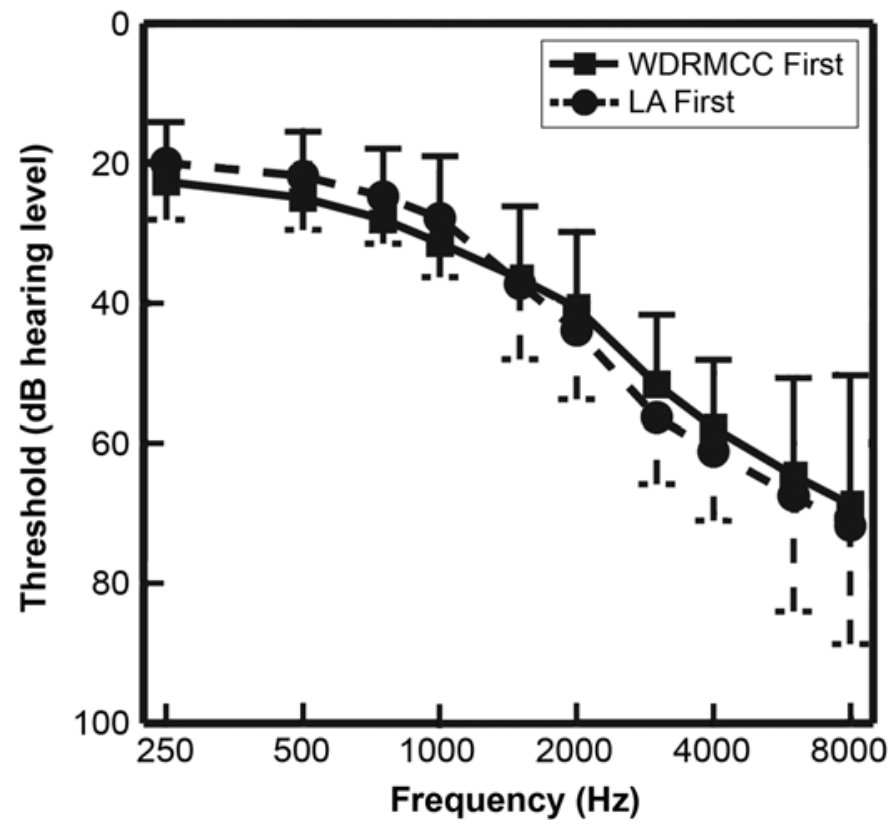

Figure 1.

Average pure tone thresholds for two groups of subjects whose first hearing aid experience was with either wide dynamic range multichannel compression (WDRMCC) or linear amplification (LA). Each average includes right- and left-ear thresholds from 19 subjects. Error bars show +1 standard deviation (SD) for WDRMCC users and -1 SD for LA users.

complexity of the experiment, age-corrected MMSE criteria were not used, which ensured that only highly functional subjects were recruited, independent of age.

In spite of the relatively strict subject selection criteria, the speech-perception performance of one subject was far below that of the other subjects. At the highest SNR used in the study (15 dB), this subject's mean correct performance was 3.8 SD below the overall mean of all subjects for speech-perception performance. Data from this subject were excluded from all figures and data analyses in this report.

HAs and batteries were provided at no cost to the subjects during their participation in the study, and the subjects were paid for the time they spent in the laboratory.

\section{Hearing Aids and Fitting}

In-the-canal (ITC) HAs from two manufacturers were used. The two HAs (Canta 730 [GN ReSound North America, Bloomington, Minnesota] and Altair ITC [Sonic Innovations, Salt Lake City, Utah]) were chosen because both were capable of WDRMCC, with a large number of independent compression channels (14 in the
Canta 730 and 9 in the Altair ITC), and because they were capable of LA, with good fits to linear targets (a result of the large numbers of channels). We used two platforms to provide generalizability of the results across more than one specific signal-processing scheme. WDRMCC with a large number of channels was used because previous results indicated that speech perception in noise improved as the number of channels increased, at least in the range of 2 to 8 channels [34,39].

Prior to any HA experience, each subject was fit bilaterally with both WDRMCC and LA fittings. The WDRMCC fitting procedure followed the manufacturer's recommendations. The LA fitting procedure used the National Acoustic Laboratory-Revised (NAL-R) target [40]. All fittings were verified with probe microphone measurements to within $5 \mathrm{~dB}$ at 500, 1000, and $2000 \mathrm{~Hz}$ and within $10 \mathrm{~dB}$ at $4000 \mathrm{~Hz}$. The mean deviation from the linear fit to the NAL-R target was $-1.1,-3.0,-2.7$, and $-5.5 \mathrm{~dB}$ at octave frequencies from 500 to $4000 \mathrm{~Hz}$, respectively. At these same frequencies, the WDRMCC fittings offered slightly less amplification at $50 \mathrm{~dB}$ sound pressure level (SPL) $(0.26,2.5,1.9$, and $0.4 \mathrm{~dB})$ and considerably less amplification at $80 \mathrm{~dB}$ SPL (5.6, 8.9, 12.7, and $14.0 \mathrm{~dB}$ ). The close match in amplification at the lower intensity indicates that the two fitting types provided approximately equal audibility for low-intensity speech information. Neither the Altair ITC nor the Canta 730 provided much amplification at $6000 \mathrm{~Hz}$ : the average measured gain was $5.8 \mathrm{~dB}$ and the average deviation from the NAL-R target was $-17.0 \mathrm{~dB}$. At $6000 \mathrm{~Hz}$, the average WDRMCC gain at $50 \mathrm{~dB}$ and the average linear gain differed by less than $0.1 \mathrm{~dB}$ and at $80 \mathrm{~dB}$ the linear gain was greater than the WDRMCC gain by $13.3 \mathrm{~dB}$. Since neither HA had a volume control, we are confident that subjects were using the prescribed gain in almost all listening situations. A reduced gain program $(-5 \mathrm{~dB})$ with the same frequency response was provided for those LA users who encountered high-intensity sound conditions, but they were instructed to use the standard LA program for all other conditions. Subjects were sufficiently satisfied with the original fittings for both WDRMCC and LA that we were able to discourage continuing adjustments. Significant or repeated fitting adjustments would have rendered a subject's data useless to the study.

As subjects entered the study, they were randomly assigned to one of two groups. For phase 1, half of the subjects used the WDRMCC fitting (4 females, 15 males, mean age $=64.9$ years) while the other half used the LA 
fitting ( 6 females, 13 males, mean age $=67.8$ years). All subjects switched from using one fitting to the other at week 32. Within each group, half received Canta 730 and half received Altair ITC HAs. The primary comparison of interest was between WDRMCC and LA fittings because we would not expect to find many within-type performance differences between the two brands.

The principal difference between the Canta 730 and the Altair ITC HAs is in the firmware implementation of the WDRMCC algorithm. The Canta 730 has a fast Fourier transform (FFT) frequency-domain processing scheme, including a reverse FFT, for generating the HA output. As a result of this FFT implementation, a $10 \mathrm{~ms}$ processing delay exists between input and output signals. The Canta 730 has 14 overlapping compression channels controlled by gain settings for 50 and $80 \mathrm{~dB}$ input levels at six frequencies $(250,500,1000,2000,4000$, and $6000 \mathrm{~Hz}$ ). The specified bandwidth of the Canta 730 is 200 to $5600 \mathrm{~Hz}$. Attack and release times are 5 and 70 ms, respectively. The Altair ITC uses a non-FFT processing scheme that produces essentially no delay between input and output signals $(<0.3 \mathrm{~ms})$. It has nine nonoverlapping half-octave compression channels that are centered at 500, 750, 1000, 1500, 2000, 3000, 4000, 6000, and $8000 \mathrm{~Hz}$. The $500 \mathrm{~Hz}$ channel is low-pass and includes the sound that would be below a $500 \mathrm{~Hz}$ halfoctave channel. The Altair ITC's compression channels are controlled by gain settings for 50 and $90 \mathrm{~dB}$ input levels at each channel center frequency. The specified bandwidth of the Altair ITC is 200 to $5700 \mathrm{~Hz}$; attack and release times are 5 and $11 \mathrm{~ms}$, respectively.

\section{Stimuli}

The stimuli used in the laboratory measurements of speech perception included six subtests (lists 3, 4, 5, 6, 8, and 10) of The City University of New York NST [41]. Previous results have shown that this combination of lists from the NST is particularly sensitive to phoneme identification differences between LA and WDRMCC signal processing [35] and phoneme identification changes over time [36]. Lists 3, 4, and 5 are consonantvowel (CV) syllables and consist of voiceless consonants (/p/, /t/, /k/, /ch/, /f/, /th/, /s/, /sh/, and /h/) followed by one of three vowels $(/ \mathrm{a} / \mathrm{/} / \mathrm{i} /$, and $/ \mathrm{u} /)$. Lists 6,8 , and 10 are vowel-consonant (VC) syllables and consist of the same three vowels followed by voiced consonants (/b/, /d/, $/ \mathrm{g} /, / \mathrm{m} /, / \mathrm{n} /$, /ng/, /v/, /TH/, and /z/). The original syllables were produced with the same male and female voice stimuli used in our previous experiments and included six exemplars of each syllable for each voice [34].

The WDRMCC and LA stimuli for each subject were digitally recorded through the subject's HAs that were programmed with the subject's individual WDRMCC and LA fittings. The recordings were done in a FONIX FC5010 sound chamber (Frye Electronic, Inc, Tigard, Oregon) with a Brüel \& Kjær (Norcross, Georgia) Type 4946 2cc coupler, Type 4134 microphone, and Type 2603 amplifier. We applied the coupler to real-ear correction to the 16-bit digital recordings before they were presented to the subjects through ER-2 earphones (Etymotic Research Inc, Elk Grove Village, Illinois). For the recordings, the syllables (at 85,75 , and $65 \mathrm{~dB}$ SPL) were mixed with speech-spectrum noise at $70 \mathrm{~dB}$ SPL, which yielded SNRs of 15,5 , and $-5 \mathrm{~dB}$. The position of the syllable in the $1.2 \mathrm{~s}$ noise varied randomly among stimuli, SNR, processing conditions, and subjects, but the syllable never extended into the initial or final $100 \mathrm{~ms}$ of the noise. For each subject, we adjusted the intensity of the stimuli recorded for testing to use the full 16-bit digitizing range for the $15 \mathrm{~dB}$ SNR LA- or WDRMCCprocessed stimuli, whichever produced the maximum HA output levels. An unaided $70 \mathrm{~dB} 1 \mathrm{kHz}$ pure tone was digitized directly at this same recording level for each subject for calibration of stimuli. The 5 and $-5 \mathrm{~dB}$ SNR recording levels were also adjusted to use the full digitizing range and digitally attenuated to correspond to the 15 dB SNR calibration tone. Auditory stimuli were presented through a 16-bit Sound Blaster Live! sound card (Creative Technology, Ltd, Milpitas, California) and amplified (RCA SA-155 integrated stereo amplifier [Thomson SA, Boulogne-Billancourt, France]) before being sent to the ER-2 earphones. We calibrated the right and left channels of the amplifier with the subject's $70 \mathrm{~dB}$ $1 \mathrm{kHz}$ calibration tones before each session. Adjusting the ER-2 earphone inputs to present the calibration tone at $70 \mathrm{~dB}$ SPL ensured that the speech stimuli were at the same level as the HA output during the recording process.

\section{Nonsense Syllable Test Procedures}

Our primary reason for prerecording the stimuli was to measure NST performance for both LA and WDRMCC stimuli for each subject throughout the entire experiment. Comparison of results for LA and WDRMCC stimuli indicates the specificity of the subjects' acclimatization to the details of the signal processing used in everyday listening. During each run of the NST, 
both LA- and WDRMCC-processed stimuli were presented in independent random order. One run consisted of one of each of the 54 syllables with LA and WDRMCC processing for a total of 108 trials. Each run included monaural presentations of one of the two voices (male or female) at one SNR to either the right or left ear.

A 2-hour laboratory session included 12 experimental runs (1,296 trials). Subjects could take breaks as needed and were encouraged to take a break of at least 5 minutes in the middle of the session. The SNRs were presented in descending order $(15,5$, and $-5 \mathrm{~dB})$ with four runs at each SNR condition, one for each voice in each ear. The order of the stimuli was independently randomized for each run. Experiments were run under computer control with generic personal computers (PCs) and Presentation ${ }^{\circledR}$ software (Neurobehavioral Systems, Inc, Albany, California).

During laboratory sessions, each subject was seated in a small sound-treated room (Industrial Acoustics Company, Bronx, New York) that was equipped with a flatpanel monitor (the secondary monitor on the experiment PC) and a serial keypad (for numerical response entry). Each trial of the experiment consisted of the following parts: (1) a numbered list of the syllables from one NST subset was displayed on the subject's monitor, (2) one stimulus from the list was presented, and (3) the subject entered the number of the syllable heard, followed by the "Enter" key to register the response. The subject's response entry was the signal for the PC to begin the next trial after a $1 \mathrm{~s}$ interval. Subjects were told that a response was required to go on to the next trial and that they should make their best guess if they were not sure of the correct response. No feedback was given. The first three sessions for each subject were devoted to learning the procedures and becoming familiar with the voices and stimuli. Two sessions included unprocessed stimuli at 15 and $5 \mathrm{~dB}$ SNR, and the third included processed stimuli at all three SNRs. Previous results indicate that the combined subjects' performance on the NST should be stable within a range of 1 percent after this amount of experience with the task, for the number of subjects, and for the number of repeated measures in the present experiment [34-36,42-45].

NST performance for each subject was measured with LA- and WDRMCC-processed stimuli before the HAs were issued (week 0) and at weeks 1, 2, 4, 8, 16, and 32 during phase 1 . Each subject completed two identical sessions at each time point. Previous power analyses indicated that data from two sessions would be needed to detect a 2 percent difference between acclimatization effects for LA and WDRMCC HAs at $p<0.05$ with 50 to 80 percent confidence in 20 to 40 subjects. Our previous experience also indicated that the consonant confusion analyses would require data from at least two sessions.

In addition to testing with LA- and WDRMCCprocessed stimuli, we measured NST performance for each subject with unprocessed stimuli (the unaided condition) at weeks 0,16 , and 32 during phase 1 . We used this unaided performance to monitor performance changes that did not depend on the acoustic properties of the processed signals.

Immediately after the phase 1 week-32 measurements were completed, the subjects' HAs were reprogrammed for the other signal processing type (LA to WDRMCC or WDRMCC to LA). The phase 1 week-32 NST data also served as the phase 2 week- 0 data for the second processing type, and NST performance was measured at weeks $1,2,4$, and 8 during phase 2 . Initially, we had planned to obtain phase 2 data at 16 and 32 weeks as well, but once it became clear that smaller performance changes were occurring after the reprogramming than in phase 1, we discontinued measurement of these last two points. Indeed, as shown in the results, the phase 1 16- and 32-week measurements failed to indicate continuing improvement. The 40 weeks of NST data track acclimatization of speech perception in four different experimental conditions: (1) new HA users with WDRMCC fittings, (2) new HA users with LA fittings, (3) HA users with 32 weeks of WDRMCC experience switched to LA, and (4) HA users with 32 weeks of LA experience switched to WDRMCC.

\section{Questionnaires}

In addition to the NST measures of speech perception, subjects also completed two questionnaires that measure aspects of HA success: the PHAB [46] and the HAPI [47]. PHAB and HAPI responses were obtained at 2,8 , and 32 weeks during phase 1 . PHAB unaided reference scores were obtained before the HAs were issued to the subjects.

\section{Data Analysis}

We performed analysis of variance (ANOVA) and Bonferroni-corrected post hoc pairwise comparisons using C Language Exploratory Analysis of Variance with Enhancements software (Herron TJ. Human Cognitive Neurophysiology Laboratory, VA NCHCS, Martinez, 
California, 2004). Kruskal-Wallis and Friedman's nonparametric tests were used to evaluate changes in the questionnaire data.

\section{RESULTS}

\section{Phoneme Perception in New Hearing Aid Users}

The fundamental questions in the present study concerned the presence of acclimatization in new HA users and whether that acclimatization would differ for WDRMCC and LA HA users. Figure 2 shows the effect of everyday HA experience outside the laboratory on NST syllable recognition in the laboratory. These results indicate that syllable recognition improved at least through the first 8 weeks of HA experience. The global ANOVA of the phase 1 data (weeks 0 to 32) confirmed that the main effect of weeks of HA experience was highly significant $\left[F_{(6,206)}=13.79, p<0.001\right]$. The only other significant main effects were expected: SNR $\left[F_{(2,68)}=1,374.2, p<0.001\right]$ and voice $\left[F_{(1,34)}=34.42\right.$, $p<0.001]$. For SNR, syllable recognition was highest at
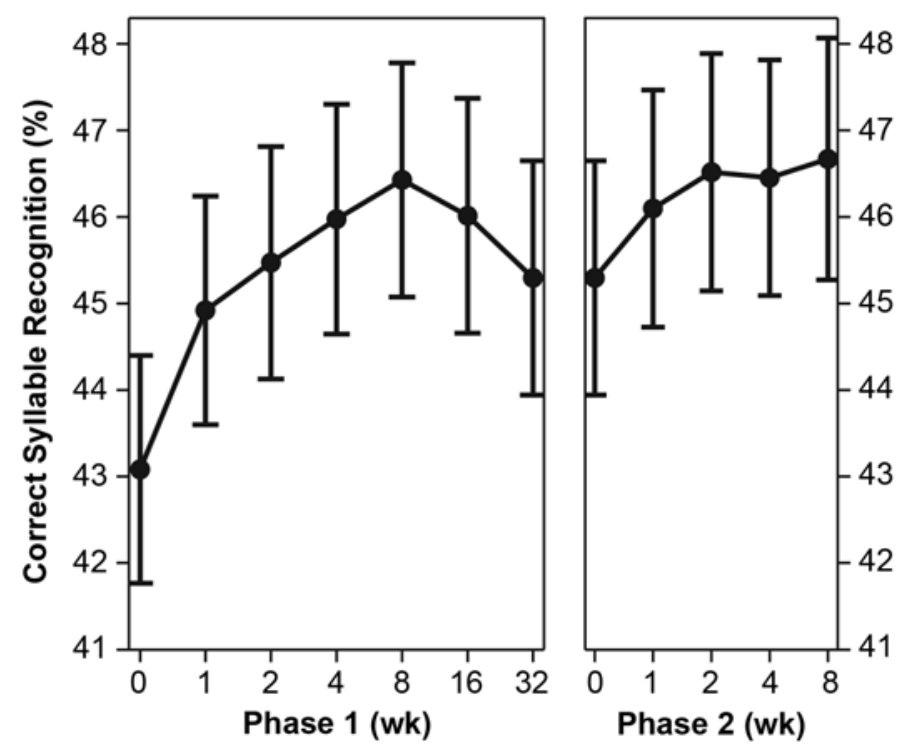

Figure 2.

Average percent correct syllable recognition on nonsense syllable tests for all subjects during first 40 weeks of normal hearing aid (HA) experience. After 32 weeks (phase 1), subjects switched HA fittings and continued normal use of HAs with new fitting. Week-32 measurement for phase 1 (left panel) also represents week-0 score for phase 2, since data were acquired immediately before algorithm switch. Error bars give standard error for each point.
$15 \mathrm{~dB}$ SNR (65.4\%), less at $5 \mathrm{~dB}$ SNR (47.7\%), and lowest at $-5 \mathrm{~dB}$ SNR (22.8\%). For voice, syllable recognition was better for the female voice (46.1\%) than for the male voice $(44.5 \%)$, as in our previous studies of WDRMCC and LA signal processing [34]. None of the other three main effects was significant: HA platform (Canta 730 or Altair ITC) $\left[F_{(1,34)}=1.164, p=0.29\right.$ ], type of HA fitting (WDRMCC or LA) $\left[F_{(1,34)}<1.0\right]$, and test stimulus signal processing (WDRMCC or LA) $\left[F_{(1,34)}=1.168, p=0.29\right]$.

Voice showed two-way interactions with three other factors. First, the recognition advantage for the female voice was greater for Canta $730(2.4 \%)$ than for Altair $\operatorname{ITC}(0.5 \%)\left[F_{(1,34)}=11.11, p=0.002\right]$. Second, the recognition advantage for the female voice was greater for LA (1.8\%) than for WDRMCC (1.3\%) test stimulus signal processing $\left[F_{(1,34)}=4.65, p=0.04\right]$. And third, the recognition advantage for the female voice was greater at 15 and $5 \mathrm{~dB}$ SNR (2.4\% and 2.7\%, respectively) than at $-5 \mathrm{~dB}$ SNR $(-0.3 \%)\left[F_{(2,68)}=9.40, p<0.001\right]$. Since these interactions were present at similar magnitudes in the phase 1 week- 0 data alone, they seem to be independent of HA experience and acclimatization and need not be considered in further detail here.

The only other significant two-way interaction was between type of HA fitting and test stimulus signal processing $\left[F_{(1,34)}=5.64, p=0.02\right]$; over the phase 1 period, subjects using LA fittings correctly recognized fewer LA than WDRMCC test stimuli ( $-0.7 \%)$, while subjects using WDRMCC fittings correctly recognized more LA than WDRMCC test stimuli (1.7\%). This interaction would be truly paradoxical if it had developed during the period of new HA experience, but it was also present at phase 1 week 0 . In phase 1 week- 0 data alone, this HA-fitting $\times$ stimulus-signal-processing interaction was very similar $\left[F_{(1,34)}=7.38, p=0.01\right]$, with subjects assigned to LA fittings recognizing fewer LA than WDRMCC test stimuli $(-0.5 \%)$ and subjects assigned to WDRMCC fittings recognizing more LA then WDRMCC test stimuli (2.3\%).

Although the main effects and interactions present at phase 1 week 0 cannot be attributed to acclimatization, the question remains of how to observe, track, and analyze the performance changes of acclimatization in the presence of these other differences. Since the essence of acclimatization is the change in speech perception relative to that observed before the subject had any HA experience, the difference between any particular performance score and the corresponding phase 1 week- 0 score should 
reflect effects of HA experience without contamination from effects and interactions that were already present before any HA experience. Performing the ANOVA on the difference data for phase 1 (weeks 1 to 32) solves another problem concerning the inclusion of phase 1 week-0 data and the data from later weeks in the same analysis: when data from phase 1 weeks 0 to 32 are used, no mechanism exits to inform the ANOVA calculation of the special role of the phase 1 week- 0 data as reference values. When difference data from phase 1 weeks 1 to 32 are used, the reference role of week 0 (phase 1 ) is incorporated into the data before it enters the ANOVA. The outcome of this difference-data ANOVA must be interpreted carefully because effects and interactions in the difference data are not the same as those in the raw-data ANOVAs of phase 1 weeks 0 to 32 or 1 to 32. For example, the expected effect of SNR must be found in both raw-data ANOVAs, but no particular SNR effect is expected in the difference data because that effect would depend on the relative improvements at $-5,5$, and $15 \mathrm{~dB}$ SNR. The remainder of the data presentation and analysis include only difference scores, where the phase 1 week- 0 scores were subtracted from later week scores individually for each subject for each condition.

\section{New Users of WDRMCC and LA Hearing Aids}

A primary hypothesis in the present research was that subjects would exhibit greater acclimatization to WDRMCC than to LA HAs. The first test of this hypothesis compared average syllable-recognition changes over 1 to 32 weeks (phase 1) for WDRMCC test stimuli in WDRMCC HA users with those for LA test stimuli in LA HA users. The WDRMCC group improved 3.74 percent and the LA group 1.71 percent, and that difference was significant (one-tailed $t=2.01, p=0.03$ ). We also had anticipated that WDRMCC users would improve more on WDRMCC test stimuli (3.74\%) than LA test stimuli (3.13\%), but that difference did not reach significance (one-tailed $t=1.38, p=0.09$ ). In the case of LA users, the small difference between improvement for LA (1.71\%) and WDRMCC (1.82\%) test stimuli was not even in the expected direction, which suggests no HAspecific change as a result of LA use.

The syllable-recognition change results for each test stimulus type for each experimental phase are shown in Figure 3. The detailed results for phase 1 (left panel of Figure 3) are entirely consistent with the overall mean results for the average syllable-recognition changes. At each point throughout phase 1, WDRMCC users scored the maximum improvement for WDRMCC test stimuli (black circles) and the second largest improvement for LA test stimuli (black squares), while LA users scored similar lower levels of improvement for both types of test stimuli. These results clearly indicate that the type of HA experience was the primary determinant of the magnitude of change over time and that the type of test stimulus signal processing had minimal effect. The trend toward an effect of test stimulus type in the WDRMCC HA users suggests some of the signal-processing specificity that might be expected in acclimatization, but no similar trend was seen for LA users.

The ANOVA of the WDRMCC HA users' difference scores (for phase 1 , weeks 1 to 32) demonstrated that

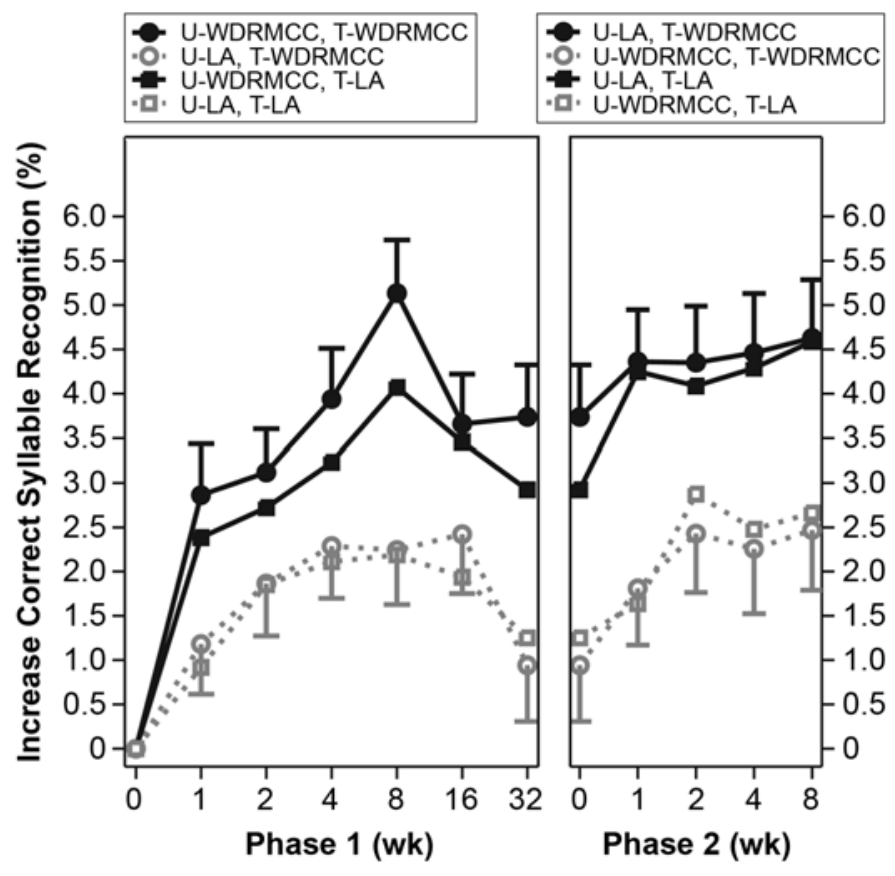

Figure 3.

Average percent correct increase for two subject groups on nonsense syllable tests showing separate results for wide dynamic range multichannel compression (WDRMCC) or linear amplification (LA) processed stimuli. U-WDRMCC or U-LA indicates algorithm used in HA outside laboratory, and T-WDRMCC or T-LA indicates test stimulus signal processing. Results normalized by subtraction of phase 1 week- 0 values; error bars are 1 standard error (SE) and are included only for T-WDRMCC curves. (SE for T-LA points were virtually the same as those for T-WDRMCC points and were omitted to facilitate visual comparisons among four conditions.) Results demonstrate that primary determinant of change in syllable discrimination was type of signal processing in initial HA experience. 
their mean syllable-recognition increase (3.44\%) was significant $\left[F_{(1,18)}=50.62, p<0.001\right]$. This performance increase also varied across week $\left[F_{(5,90)}=2.74, p=\right.$ $0.02]$. Post hoc pairwise comparisons found a significant difference between weeks 1 and $8(p<0.05$, Bonferroni corrected to 0.003), which suggested that performance improvements continued through week 8. The WDRMCC users' data in Figure 3 support this conclusion, showing monotonic increases for both WDRMCC and LA test stimuli during weeks 0 to 8 . Although the performance for weeks 16 and 32 was not significantly less than week 8 , this reduction suggests that some other factor might be interfering with measurement of further performance increases, and we will return to this issue in the "Discussion" section. The ANOVA for the WDRMCC HA users showed no other main effects or interactions.

The ANOVA of the LA HA users' difference scores (for phase 1 , weeks 1 to 32) indicated that their mean syllable-recognition increase (1.77\%) was significant $\left[F_{(1,18)}=5.46, p=0.03\right]$. A significant change was also noted across week $\left[F_{(5,90)}=2.93, p=0.02\right]$, but post hoc pairwise comparisons found no significant differences. The LA HA users' data in Figure 3 suggest that all the performance gain was completed prior to week 4 . The results for weeks 4 to 16 seem to define a plateau level about 2 percent, but week 32 results suggest reduced performance similar to weeks 16 and 32 for WDRMCC HA users. The ANOVA for the LA HA users showed no other main effects or interactions.

\section{Phoneme Perception After Switching Hearing Aids}

At 32 weeks (end of phase 1), subjects switched from WDRMCC to LA or from LA to WDRMCC HA fittings. The syllable-recognition results after the switch are shown in the right panels of Figures 2 and 3. Recall that no time had elapsed between phase 1 week 32 in the left panels and phase 2 week 0 in the right panels because those data points represent the same data taken immediately before the HA fittings were switched. Data after the switch can be examined from two different perspectives: (1) as a simple extension of the data before the switch or (2) as a distinct time period with no reference to the earlier data before the switch. The pattern of results seen in Figure 2 suggests that the first perspective is more appropriate because it is primarily the phase 1 week-32 performance that fell well below the increasing asymptotic-performance curve defined by the other points. Indeed, without phase 1 week 16 , week 32 (phase 2 week 0 ), and phase 2 week 1 , an almost perfect continuity of performance across phases would exist (Figures 2 and 3). Of course, the only way to have demonstrated that this pattern of results did not depend on the switch of HA algorithms would have been to include control groups on the same testing schedule, but without a genuine switch in HA signal-processing algorithm.

Next we will examine the phase 2 data from the second perspective, as a distinct period for observing the effects of the change in algorithm on syllable recognition. The average NST performance increase for phase 2 weeks 1 to 8 (relative to phase 2 week 0 ) was 1.23 percent for WDRMCC use after 32 weeks of LA use and 1.05 percent for LA use after 32 weeks of WDRMCC use; that difference was not significant $(t=0.365, p=0.4)$.

In the ANOVA for the group that switched from LA to WDRMCC (Figure 3, right panel, gray curves), a significant change was noted in phoneme discrimination across weeks $\left[F_{(4,72)}=4.69, p=0.002\right]$, and pairwise comparisons $(p=0.05$, Bonferroni corrected $p=0.005)$ indicated that phase 2 weeks 2, 4, and 8 were different from phase 2 week 0 . For the group that switched from WDRMCC to LA (Figure 3, right panel, black curves), the change across weeks was also significant $\left[F_{(4,72)}=\right.$ $2.55, p=0.046]$, but in pairwise comparisons only phase 2 weeks 0 and 8 differed. Although significant performance changes were noted for phase 2, these results indicate, at most, a small acclimatization effect that may be a little larger for WDRMCC (1.23\%) than for LA (1.05\%) experience; neither effect was of comparable magnitude to that seen for initial WDRMCC experience (3.44\%), which was significantly larger than the effect of initial LA experience (1.77\%). Clearly, WDRMCC experience after the switch does not have the same effect as initial WDRMCC experience.

\section{Changes in Patterns of Consonant Confusions}

The NST was constructed to facilitate consonant confusion analyses, in that the most commonly confused consonants are segregated into separate lists. Patterns in changes of consonant confusions on the NST can provide information about the explanations for the changes. The consonant confusion matrices for the two subject groups for phase 1 week 0 (before any HA experience) are given in Figure 4. In Figure 4, the stimulus is on the abscissa, the response is on the ordinate, and the area of the circles represents the number of responses that were given for each stimulus. Perfect performance would yield large circles 
along the positive diagonal and none anywhere else. Random performance would yield a uniform distribution of small circles throughout the matrices. The consonants are grouped by manner along the axes as follows: for the voiced VC syllables (Figure 4 (a) and (c)), the plosives are first, followed by the nasals, and finally the fricatives. A prevalence of within-manner errors can be seen clearly in these results, most strongly for the nasal consonants, where all the off-diagonal circles in the central $3 \times 3$ syllable square are larger than any to the left (nasal responses to plosive stimuli) or the right (nasal responses to fricative stimuli). A response bias for $/ \mathrm{TH} /$ and against $/ \mathrm{v} /$ is also apparent in the horizontal rows corresponding to those responses. For the voiceless CV syllables (Figure 4 (b) and (d)), relatively few /th/ responses were noted, with /f/ being the most common response for the voiceless /th/. The /f/-/th/ confusion is not unusual for people with normal or impaired hearing [34-35,48-50].

The difference between phase 1 week- 0 and week- 8 confusion matrices is shown in Figure 5. Response
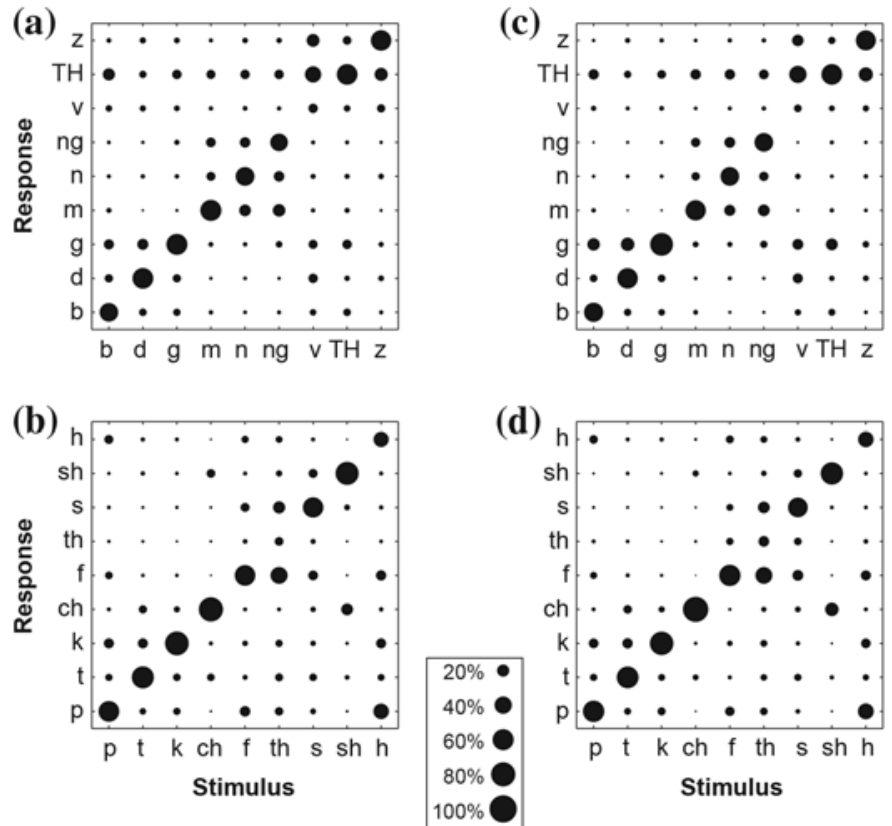

Figure 4.

Confusion matrices before any hearing aid (HA) experience for subjects whose first HA experience was with wide dynamic range multichannel compression for (a) vowel-consonant (VC) syllables and (b) consonantvowel syllables (CV) and for subjects whose first HA experience was with linear amplification for (c) VC syllables and (d) CV syllables. Area of circles represents number of responses for that stimulus-response combination. increases are represented by black circles; response decreases are represented by gray circles. As in Figure 4, the area of the circle represents the magnitude, but in this case it is the magnitude of the difference between week-0 and week- 8 (phase 1 ) response. The more consistent pattern of larger black circles on the positive diagonal for the WDRMCC-experience panels (Figure 5 (a) and (b)) represents the greater performance improvement for the WDRMCC- versus the LA-experience group, as seen in Figures 2 and 3. However, the changing error patterns are of greater interest here.

In the phase 1 week- 0 voiced VC confusion matrices of Figure 4, we noted the response bias for /TH/ and against $/ \mathrm{v} /$. For the LA-experience group, a shift in that response bias is seen: /TH/ responses decreased for all stimuli (all gray circles in that row) and $/ \mathrm{v} /$ responses increased for all stimuli (all black circles in that row) (Figure 5(c)). A similar shift in response pattern is seen in this group, away from $/ \mathrm{g} /$ responses (all gray circles in that row). The voiceless CV syllables LA-experience
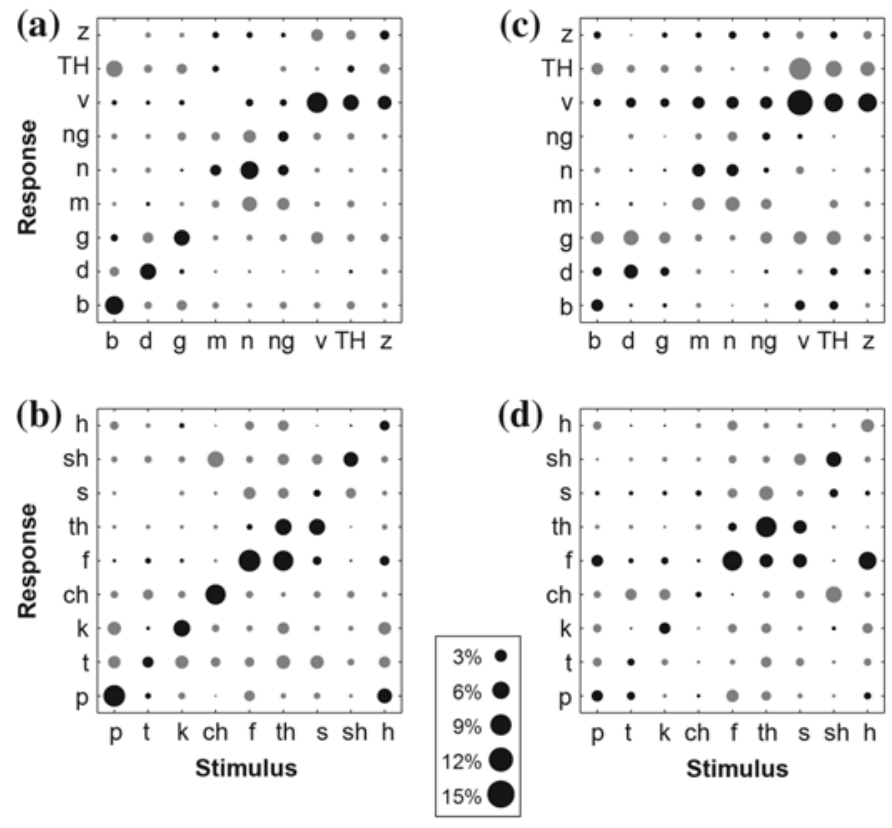

Figure 5.

Change in confusion matrices after first 8 weeks of hearing aid experience (phase 1) for (a) wide dynamic range multichannel compression (WDRMCC) users for vowel-consonant (VC) syllables, (b) WDRMCC users for consonant-vowel (CV) syllables, (c) linear amplification (LA) users for VC syllables, and (d) LA users for CV syllables. Gray circles indicate fewer and black circles indicate more responses after 8 weeks. Area of circles represents change in number of responses for that stimulus-response combination. 
confusion matrix shows decreased /h/ and increased /f/ responses (Figure 5(d)). Such patterns of response shifts that are somewhat independent of the stimulus presented suggest that the subjects may have changed response biases rather than learned to recognize the stimuli.

For the WDRMCC-experience group, similar general patterns of response shifts are absent (Figure 5(a)). The /v/ response row includes black and gray circles, but the circles representing manner confusions are quite small (absent for $/ \mathrm{m} /$ where no change was noted). Furthermore, the gain in $/ \mathrm{v} /$ responses was not just a loss of $/ \mathrm{TH} /$ responses, but an increase of correct $/ \mathrm{TH} /$ responses as well as a small increase in correct-manner $/ \mathrm{TH} /$ responses to $/ \mathrm{v} /$ stimuli. Similarly, a smaller number of $/ \mathrm{m} /$ responses were noted, particularly to $/ \mathrm{n} / \mathrm{and} / \mathrm{ng} /$ stimuli, without much reduction in correct $/ \mathrm{m} /$ responses. An increase in correct responses to /g/ stimuli was also noted as well as an increase in manner-correct responses to /b/ stimuli.

The confusion difference matrices for the smaller performance changes that occurred after the switch showed no clear patterns for either group and will not be presented or discussed further. Of course, given the small size of the changes in the phoneme-discrimination scores, we are not surprised that little was found in the confusion analyses.

\section{Unprocessed Stimuli}

In addition to the NST results for LA and WDRMCC stimuli, results were obtained for unprocessed stimuli at weeks 0,16 , and 32 of phase 1 . Unprocessed stimuli in this context are equivalent to the unaided condition of other studies. Since only 15 and 5 dB SNRs were used in the unprocessed-stimulus sessions, only 15 and $5 \mathrm{~dB}$ SNR processed-stimuli results were included in comparison analyses. In the combined ANOVA for the LA- and WDRMCC-experience groups, subjects had discrimination scores that were 15 percent poorer for unprocessed than for processed stimuli $\left[F_{(2,72)}=118.65, p<0.0001\right]$. Post hoc testing showed that LA and WDRMCC test stimuli both differed from unprocessed test stimuli $(p<$ 0.0001, including Bonferroni correction) but did not differ from each other. This result is not surprising but does demonstrate that the two fittings were approximately equally good for speech perception at moderately loud intensities in noise.

In separate ANOVAs for WDRMCC and LA users, each including phase 1 week-16 and -32 difference scores for all three types of test stimuli (unprocessed, LA- and WDRMCC-processed), the mean performance change was significant for both WDRMCC $\left[F_{(1,18)}=44.19, p<\right.$ $0.0001]$ and LA $\left[F_{(1,18)}=4.74, p=0.04\right]$ HA groups. The only other significant effect was for test stimulus signalprocessing algorithm in WDRMCC users $\left[F_{(2,36)}=5.39\right.$, $p=0.009]$, where the gain for unprocessed stimuli was significantly smaller in post hoc pairwise comparisons. These results for WDRMCC and LA users are shown in Figure 6. Previous figures (Figure 2 and 3) already had illustrated the performance differences between WDRMCC and LA experience, but these results demonstrate that LA users showed the same performance gain for unprocessed stimuli as they did for WDRMCC- and LA-processed test stimuli. Furthermore, the LA users' gain on any type of test stimulus is at the same level as the WDRMCC users' gain on unprocessed test stimuli. These results indicate that the LA users' experience outside of the laboratory did not help them use the additional information provided in the LA and WDRMCC test stimuli because they could not recognize these processed stimuli any better than the unprocessed test stimuli. In contrast, WDRMCC users' experience outside of the laboratory did help them use the additional information in the LA and WDRMCC test stimuli. The WDRMCC users' results are critical to the interpretation of the LA users' results because they demonstrate that both LA and WDRMCC test stimuli have additional information in the conditions used in these experiments. A primary topic for discussion will be the differences between WDRMCC and LA experience that might account for these results.

\section{Questionnaires}

Figure 7 shows the results for the HAPI and PHAB questionnaires during phase 1 of LA and WDRMCC use. Both questionnaires indicate that LA and WDRMCC fittings were subjectively successful. A trend toward greater subjective success for the LA fittings can be seen in Figure 7 (except in the PHAB aversiveness subscale, where the trend was for less aversiveness for WDRMCC), but none of the differences between the groups of LA and WDRMCC users was significant in Kruskal-Wallis tests for either questionnaire (at phase 1 week 2, 8, 32, or all three combined).

Clearly, the questionnaires were not very sensitive to the improvement in syllable discrimination found in the NST results. The HAPI showed a virtually flat subjective performance trend over time, and the PHAB showed a slightly decreasing subjective benefit trend over the same time. Differences across phase 1 weeks 2 to 32 were analyzed with Friedman's tests for global scores and subtests 


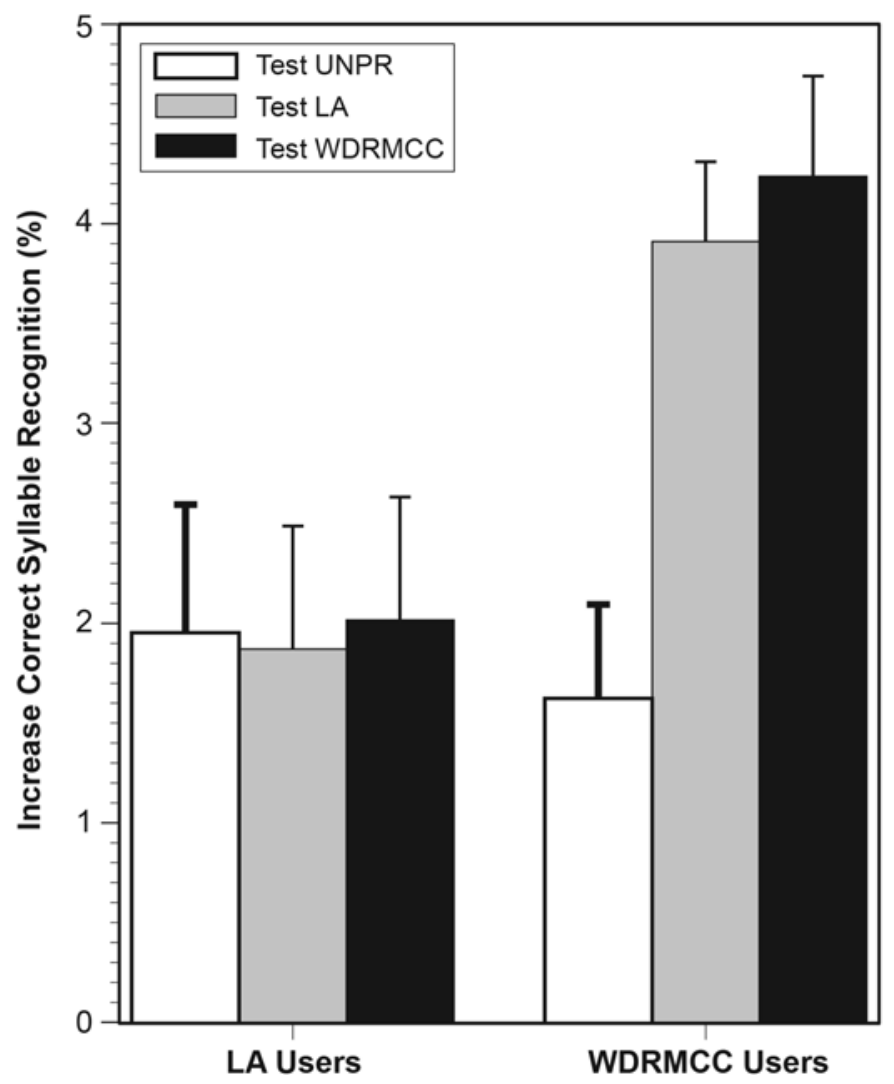

Figure 6.

Performance improvement for three types of test stimuli (unprocessed [UNPR], linear amplification [LA], and wide dynamic range multichannel compression [WDRMCC]) averaged across 16 and 32 weeks of LA or WDRMCC use. Error bars show 1 standard error.

of the HAPI and PHAB questionnaires for LA and WDRMCC user groups, separately and combined. No significant differences were found $(p<0.01$ because of large number of tests).

The reader should not be concerned about the negative values seen for the aversiveness and distortion subscales on the PHAB. We would be surprised if some tendency toward increased aversiveness did not accompany the amplification of sound provided by the new HA. The lack of significantly greater distortion for WDRMCC compared with LA fittings suggests that the WDRMCC signal processing is not perceived as particularly distorting.

\section{DISCUSSION}

\section{Critical Components for Producing Acclimatization}

Two critical components were necessary for producing acclimatization in the present study: the subjects had
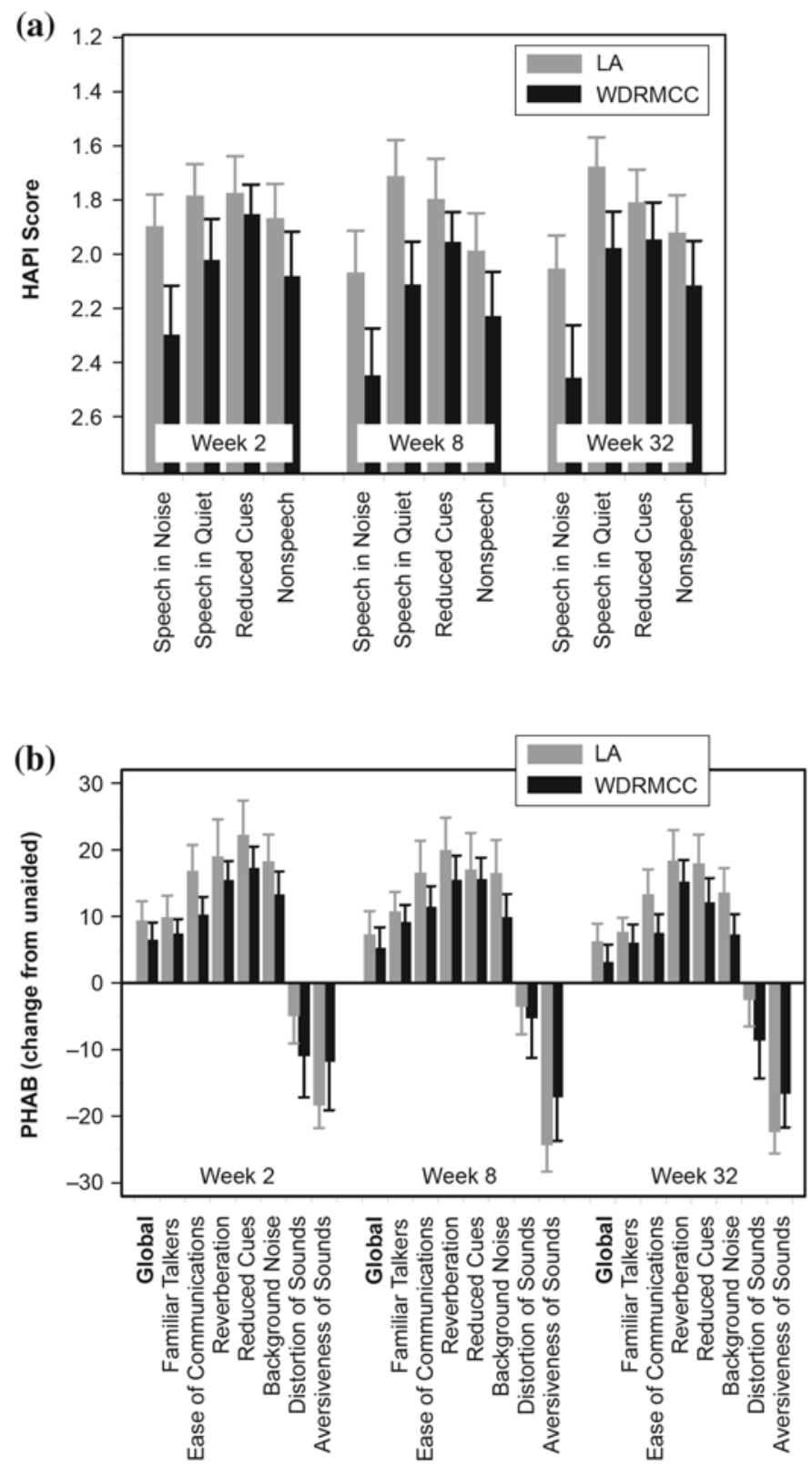

Figure 7.

Results at 2, 8, and 32 weeks (phase 1) of hearing aid (HA) experience for (a) Hearing Aid Performance Inventory (HAPI) questionnaire and (b) Profile of Hearing Aid Benefit (PHAB) questionnaire. Error bars indicate 1 standard error (SE). Scale for HAPI score is inverted because lower values on that scale indicate better subjective performance; values 3.0 indicate that HA helps and 1.0 ("very helpful") is top of HAPI scale. On PHAB, values plotted are changes from unaided scores: larger positive magnitudes or smaller negative magnitudes indicate greater subjective benefit. As expected from magnitude of SEs, none of differences between linear amplification (LA)- and wide dynamic range multichannel compression (WDRMCC)-experience groups, nor changes over time, was significant. 
to be new HA users and their HAs had to provide WDRMCC signal processing. Only WDRMCC experience, without prior LA experience, produced clear evidence of acclimatization.

Multiple aspects of the results combine to indicate that acclimatization occurred only in new WDRMCC HA users:

1. The magnitude of improvement in the NST was greater for subjects who initially used WDRMCC HAs than subjects who initially used LA HAs.

2. The improvement occurred over a longer period of time for the WDRMCC- than for the LA-experience group.

3. The patterns of change in the confusion matrices for the two groups differed. The LA group's pattern suggested changes in response strategy, while the WDRMCC group's pattern indicated specific improvement in phoneme discrimination.

4. The performance improvement over time for the unprocessed stimuli was the same in the WDRMCC and LA groups, while the performance improvement over that same time for processed stimuli was greater in the WDRMCC group. In contrast, the LA group showed no more improvement for the processed than for the unprocessed stimuli.

The improvement that occurred in both groups for unprocessed stimuli cannot represent acclimatization because all the subjects began the study with no previous HA experience; i.e., they began the study thoroughly "acclimatized" to unprocessed stimuli because they had heard nothing but unprocessed speech before the study. Improvement on unprocessed stimuli must be attributed to subjects' increased skill at performing the NST, i.e., to procedural learning. Acclimatization must be seen as a performance improvement for processed stimuli, and that improvement must be greater than the nonspecific improvement seen for unprocessed stimuli. From this perspective, the LAexperience group showed no evidence of acclimatization to the new HA. In contrast, the WDRMCC-experience group did show greater improvement on processed than on unprocessed stimuli, which yielded clear evidence of acclimatization. Finding acclimatization in the WDRMCC group also reinforces the lack of acclimatization for the LAexperience group by validating the methods. If LA experience produced acclimatization comparable to that of WDRMCC experience, then that LA acclimatization should have been found. Any acclimatization that may have occurred with LA must have been so much less than that which occurred with WDRMCC that we could not detect it in the presence of the procedural learning.

\section{Explanation for Acclimatization with WDRMCC but not LA}

Any explanation for the acclimatization effects reported here must answer three major questions: (1) Do any factors exist other than the nature of the signal processing that can account for the difference in acclimatization between new users of LA and WDRMCC HAs? (2) Why were the results within the LA- and WDRMCC-experience groups so similar for LA- and WDRMCC-processed stimuli? (3) How can we explain that new users of WDRMCC HAs learned to take advantage of information in processed syllables when new users of LA HAs did not?

Although a complete answer to the first question is impossible because we cannot rule out all other factors that may have spuriously caused a result, a description of how the experimental design has dealt with other factors is important. Many factors can be considered under the general topic of audibility: speech outside the laboratory, as well as test syllables in the laboratory, should have essentially equal audibility ${ }^{*}$ for the LA and WDRMCC HA fittings. Using the same HA platforms for the LA and WDRMCC fittings helped us ensure equal audibility because both fittings were limited by the same technical specifications. Using two different platforms also helped us because any unknown problem for one type of fitting on one platform is unlikely to occur for the other. Furthermore, as noted in the description of fittings in the "Methods" section, fittings were verified with probe microphone measurements. The findings reported there, that the fit to the NAL-R target was good and that the LA fitting offered slightly more amplification at $50 \mathrm{~dB}$ SPL input than the WDRMCC fitting, indicate that audibility

\footnotetext{
*Two such different signal-processing algorithms cannot produce entirely equal audibility across the broad range of sound conditions that HA users will encounter outside the laboratory. The capacity of WDRMCC to adjust the amplification independently in different frequency channels indicates that the audibility of a low-intensity component at one frequency, in the presence of high-intensity components at other frequencies, may be enhanced in WDRMCC signal processing. Even if the two algorithms amplified the lowintensity component identically (as expected for "equal audibility" fittings), WDRMCC's reduced amplification of the high-intensity components might reduce masking of the low-intensity component for WDRMCC relative to the masking for LA. Of course, such audibility differences that are from the nature of the algorithms, rather than fitting differences, would not justify a claim of unequal audibility.
} 
for LA and WDRMCC was essentially the same, and thus, that audibility differences cannot explain the differences in acclimatization that were found. The LA and WDRMCC test syllable results from phase 1 week 0 also support this conclusion, since no significant syllablerecognition difference was found between LA and WDRMCC test stimuli. However, if conventional audibility considerations cannot explain these acclimatization results, then other critical differences between LA and WDRMCC HAs must exist that can.

The second question listed above must be answered because the result, that LA- and WDRMCC-processed stimuli failed to differentiate between acclimatization effects for the LA- and WDRMCC-experience groups, contradicts an implicit assumption in the experimental design. Independent of how well the two types of stimuli were discriminated by subjects with no HA experience, a subject's experience with one type of HA might be expected to produce greater improvement for the stimuli recorded through that type of HA. The result that LA and WDRMCC stimuli showed similar performance changes in both groups indicates that the acclimatization effect does not correspond to highly specific learning of the detailed effects of the signalprocessing algorithm. From the general perspective of speech perception, this lack of specificity to fine details of stimuli may not be surprising because speech phoneme identification is maintained across large variations in the speech signal. Furthermore, similar speech discrimination with LA and WDRMCC signal processing in a limited range of stimulus conditions is not new, and considering previous explanations for such results in the present context will be useful.

A pioneering study of WDRMCC included extensive discussion of this issue [39], which also has been recognized in more recent studies [34-35,51-52]. When Barfod found that speech perception with 4-channel WDRMCC (his maximum) was as good as that with LA, he attributed this equality of performance to the limited range of intensities and the precise adjustment of the LA processing in his experiment. Virtually all the speech information available to normal-hearing subjects should have been available to the hearing-impaired subjects in his LA- and WDRMCC-processed stimuli. If the same is true of the stimuli in the present experiment, i.e., that essentially the same array of normal speech cues is available in both sets of stimuli, then both sets of stimuli should produce the same results. Furthermore, these results should be affected by changes in the subjects' use of speech cues whether the changes were caused by acclimatization to the corresponding HA or by another factor.

Although LA- and WDRMCC-experience groups showed similar results for the different stimulus types, only the results of the WDRMCC group demonstrated that the stimuli contained the acoustic information they needed to improve phoneme discrimination. The third question remains: Why was there acclimatization with WDRMCC but not LA experience? Here again, Barfod's discussion offers insight [39]. He argued that, although WDRMCC could not be expected to produce better performance than LA under his limited test conditions, WDRMCC would be a better HA in the unlimited conditions outside of the laboratory because it would adjust to all speech and noise intensities and also to different noise spectra. Independent of the sound intensity at various frequencies, WDRMCC could present a more consistent sound environment to the HA user. In the ideal case, any sound component that was audible to a normal-hearing individual would also be audible to a HA user. In a lessthan-ideal case, some normally audible components might be inaudible because of insufficient amplification or increased masking at higher intensities. In either case, however, the mapping of the salience and/or loudness of the various frequency components of sounds would be consistent with the normal-hearing experience, even if it did not match the normal experience in precise detail.

In contrast to WDRMCC signal processing, LA signal processing could not produce such a consistent experience of the normal range of sound intensities. Without compression, only a smaller range of intensities can be accommodated at each frequency between the perception threshold and uncomfortable loudness (the subject's remaining dynamic intensity range). Furthermore, unless the frequency response of the amplification continuously adjusted to the sound environment as it does in WDRMCC, the intensity range available at any one frequency would depend on the intensities at other frequencies because the volume control (automatic, manual, or fixed at fitting) must be set to limit discomfort from excessive loudness at any frequency.

From this perspective, WDRMCC HAs provide a much more consistent experience of the auditory information available in aided speech. Consider the fundamental idea of WDRMCC: to transform as much as possible of the frequency-intensity space of normal hearing into the residual frequency-intensity space remaining for the hearing-impaired individual. In this process, low intensities of normal frequency-intensity space are amplified to the low intensities of impaired space, moderate 
intensities of normal space are amplified to moderate intensities of impaired space, and high intensities of normal space are amplified to high intensities of impaired space. The amplification must be compressive because more amplification is required at low intensities and little or none at high intensities, and the compression must vary with frequency because impaired thresholds, and thus the remaining intensity ranges, vary with frequency. In ideal WDRMCC signal processing, all the information present in the normal frequency-intensity space would be available in the impaired frequency-intensity space and an intensity change in normal space would always correspond to a smaller intensity change in the same direction in impaired space. Although one could argue that LA would produce a similarly consistent mapping, this mapping would be restricted to a reduced range of input intensities; either lower normal intensities that cannot be amplified to impaired thresholds or higher normal intensities that will be amplified so much that discomfort and/or distortion will limit their utility. Furthermore, greater amplification of high-intensity components in LA versus WDRMCC could cause greater masking of lowintensity components for LA versus WDRMCC users, and such masking would occur in LA and not WDRMCC users even if the amplification of the low-intensity components was the same in LA and WDRMCC HAs.

Our hypothesis is that WDRMCC's consistent transformation of normal frequency-intensity space facilitated acclimatization. As long as the frequency channels are not too broad and the time constants not too slow, each frequency component of speech information can be amplified to consistent relative intensities, independent of the sound energy at other frequencies or times. As a result, the WDRMCC HA user will hear the frequency components of

\footnotetext{
*Precise definitions of too broad and too slow are yet to be determined, but the capability of WDRMCC to amplify any frequency component appropriately for its intensity depends on that component being the highest-intensity component in its frequency channel and on the lack of a previously present higher-intensity component that is still controlling amplification in the channel. Any higher-intensity component controlling amplification in the same channel will determine the level of amplification at a less-than-appropriate level for the less-intense component. WDRMCC processing with larger numbers of independent, nonoverlapping frequency channels and rapid time constants will perform best in this respect. In WDRMCC systems with only a few channels, a fortunate few frequency components determine amplification for all others and often cause inappropriate variation of the intensities of these other components. Slow time constants lead to similar problems with sequential components.
}

speech, information that would not be available to the LA HA user under the same conditions. One such frequency component would be a low-intensity, high-frequency component occurring simultaneously with higher-intensity, lower-frequency components. WDRMCC would provide relatively greater amplification for the high-frequency component than the low-frequency component and thus bring the high-frequency component above threshold in the presence of the lower-frequency components. Of course, this speech information would also be available to the person with normal hearing, and in this sense, the WDRMCC HA user gets a more consistent representation of normal speech information than is possible for the LA HA user.

\section{Acclimatization and Perceptual Learning}

Acclimatization reflects spontaneous perceptual learning that occurs as a result of the HA user's everyday acoustic experience. In that context, the performance improvements found here are interesting because they resemble those found with perceptual training [53]. Acclimatization and perceptual training produced similar changes in the confusion matrices as well as similar interactions with SNR. The improvements in syllable recognition found for perceptual training were larger than the benefits found here for acclimatization, which possibly reflects that the intensive training conditions needed for optimal perceptual learning [54-55] rarely occur in normal experience. Furthermore, perceptual training was effective in experienced as well as new HA users. Perceptual training may accelerate acclimatization in new HA users and maximize HA performance in both new and experienced HA users.

\section{Comparison with Previous Studies of Acclimatization}

The present results are consistent with the pattern of previous results in suggesting that clear evidence for acclimatization may be quite elusive [1,22-23]. In the present study, acclimatization was found in one of four conditions, i.e., only for new users of WDRMCC HAs. In contrast, we found no acclimatization for new users of LA HAs or after 32 weeks when users switched from one HA to the other (WDRMCC to LA or LA to WDRMCC). Since most previous positive and negative acclimatization results have been obtained with HAs similar to the LA fitting used here, our result for new LA users adds one more negative result to this count.

Two other studies have reported acclimatization in experienced HA users who switched to different HAs [10,14]. Although these studies provided new HAs rather 
than just a change of programming as in the present study, whether they provided a greater acoustic change than our programming switch is unclear. Of course, the lack of acclimatization after the switch from WDRMCC to LA is consistent with the similar lack of acclimatization for new LA users. The lack of acclimatization after the LA to WDRMCC switch suggests that prior LA experience may have inhibited the acclimatization that occurred for new WDRMCC users. We hypothesized above that consistent new speech cues initiated the acclimatization in new WDRMCC users and that the inconsistency of these cues in LA experience limited acclimatization. Inconsistent stimulus experience would be expected to reduce the opportunity for neuroplastic changes in speech perception. Perhaps the inconsistent experience of cues in LA continues to limit the incorporation of the cues into speech perception, in spite of the consistency provided by WDRMCC after the switch. The existence of a critical period for acclimatization seems unlikely, but longer WDRMCC experience or specific training in consonant perception with WDRMCC might be needed for users to overcome the inhibitory effects of LA experience.

Although most of the previous positive and negative acclimatization results have been obtained with standard linear fittings, our results in new HA users are consistent with suggestions that more complex HAs may show greater acclimatization [56-57]. A follow-up study of digital HA users found improved speech recognition in noise at 1 year compared with 1 month [13], which is consistent with our WDRMCC results that acclimatization continued beyond 4 weeks. Severe-to-profound hearing-impaired subjects also showed speech recognition improvements over at least 4 weeks after they switched to digital nonlinear power HAs [19]. In contrast, a study that compared different fittings for 2-channel compression HAs showed no acclimatization after 4 weeks of field experience [8]. Of course, none of the HAs in these studies would provide the same type of consistent intensity transformation discussed for WDRMCC, but the nature of acclimatization may vary for different types of HAs and degrees of hearing loss. In subjects with severe-to-profound hearing loss [19], the degree of loss limits the amount of speech information that can be provided because of the severely reduced residual dynamic intensity range and deficits in the spectral and temporal acuity of the remaining hearing. Acclimatization in severeto-profound loss must operate within this limited information set.

\section{Postswitch Acclimatization and Performance Before Switch}

The possibility of reduced NST scores for the last measurement points in the phase 132 -week acclimatization was already mentioned briefly in discussing the results for the 8 weeks (phase 2) after the switch in HA signal-processing algorithms. The clear possibility of a performance reduction before the switch complicates interpretation of performance after the switch: Is improvement after the switch merely recovery, or is it acclimatization to the other HA? In either case, the small magnitude of the postswitch improvement means that the postswitch acclimatization, if it is present at all, is quite small relative to the initial WDRMCC HA acclimatization.

The combined data in Figure 2 clearly indicate performance improvements during the first 8 weeks of phase 1 , but then suggest a 1 percent performance decrement, which is fully recovered by the second week of phase 2 . In the four different conditions of HA use and test stimuli signal processing in Figure 3, the 1 percent decrement between week 8 and week 32 (phase 1) was present for all four conditions although the relative position of the phase 1 week 16 performance varied across conditions. That this performance decrement was due to setback in acclimatization seems unlikely, and such an explanation would also predict a larger decrement for subjects using WDRMCC than LA HAs. Indeed, little or no decrement would be seen in LA users because they showed little or no acclimatization.

At least two possible explanations exist for the relatively poor performance at weeks 16 and 32 of phase 1 that would be consistent with equal decrements for LA and WDRMCC groups. First, the subjects were returning from 8 to 16 weeks away from testing. Although these subjects had considerable practice at this relatively easy task during the earlier part of the experiment, they may have needed additional practice to return to optimal performance after that much time away from the task. Second, at least in the case of phase 1 week 32, subjects may have experienced negative psychological effects of having to return to the complex and time-consuming experimental schedule required immediately after the switch. A negative psychological response to returning to the experimental schedule, however, might be expected to carry over into one or more weeks of phase 2 . The relatively quick performance recovery, predominantly in the first week of phase 2, supports the first alternative. 
If the performance decrease from weeks 8 to 32 of phase 1 was due to a loss of NST procedural skills, any conclusion we can make about the duration of acclimatization for WDRMCC HAs would be limited. The present results indicate that acclimatization continued for at least 8 week but do not provide good evidence for when, if ever, it was completed. The data from weeks 2 to 8 of phase 2 suggest the possibility of continuing improvement, but the complexities of the difference between acclimatization for LA and WDRMCC, the switch of HAs, and the performance decrement prior to the switch should be sufficient to inhibit further speculation along these lines.

\section{CONCLUSIONS}

This study measured acclimatization under four different conditions: (1) WDRMCC fittings in new HA users, (2) LA fittings in new HA users, (3) LA fittings after 32 weeks of WDRMCC experience, and (4) WDRMCC fittings after 32 weeks of LA experience. Although two questionnaires (PHAB, HAPI) showed no significant improvements over time, the NST in speech-spectrum noise provided clear evidence of acclimatization for WDRMCC fittings in new HA users during the first 8 weeks of HA experience. In NST testing during WDRMCC experience, the magnitude of performance gain from acclimatization was 45 percent of the performance gain from HA use. The small performance gains seen in new HA users with LA fittings were not consistent with acclimatization, and the smaller changes at the end of phase 1 were too small for further analysis.

To explain the presence of acclimatization in the WDRMCC-experience group and its absence in the LAexperience group, we must find a large difference between the auditory experience with WDRMCC and LA fittings. We attributed this difference in auditory experience to the more consistent encoding of intensity across frequency and intensity in WDRMCC versus LA processing. Only WDRMCC processing with many independent frequency channels and short time constants can consistently encode virtually the entire normal-hearing frequency-intensity space into the frequency-intensity space still available to the individual with mild-to-moderatelysevere hearing loss. Given this more consistent encoding of the daily sound environment, the individual can rely on more normal speech cues and acclimatization can be measured as improved consonant identification on the NST. The lack of acclimatization for WDRMCC experi- ence following 32 weeks of LA experience suggests that LA experience limits subjects' ability to switch to using more normal speech cues even after LA has been replaced by WDRMCC experience.

The greater acclimatization for WDRMCC complicates direct comparisons of the efficacies for WDRMCC and LA HAs. LA that would be equivalent to WDRMCC on the NST immediately after fitting would show less than 80 percent of the benefit of the same WDRMCC HA after acclimatization.

\section{ACKNOWLEDGMENTS}

We would like to thank the VA NCHCS Audiology Service for assistance in recruiting subjects and for the use of its facilities for HA fitting. We would also like to thank David L. Woods, G. Christopher Stecker, and Timothy J. Herron for useful and stimulating discussions, as well as comments on earlier versions of this article.

Dr. Roup is now with the Department of Speech and Hearing Sciences, The Ohio State University, Columbus, Ohio.

This material was based on work supported by the VA Rehabilitation Research and Development Service, grant C2551R, and the National Institutes of Health/ National Institute on Deafness and Other Communication Disorders, grant DC005814. Dr. Simon was also supported by the National Institute on Disability and Rehabilitation Research, grant H133G030118, and The SmithKettlewell Eye Research Institute. GN Resound (Chicago, IL) and Sonic Innovations (Salt Lake City, UT) provided their hearing aids and the necessary fitting hardware and software at no cost to the study. Neither GN Resound nor Sonic Innovations had any involvement in the study design; data collection, analysis, or interpretation; or writing or submission of this article.

The authors have declared that no competing interests exist.

\section{REFERENCES}

1. Turner CW, Humes LE, Bentler RA, Cox RM. A review of past research on changes in hearing aid benefit over time. Ear Hear. 1996;17(3 Suppl):14S-28. [PMID: 8807271]

2. Bentler RA, Niebuhr DP, Getta JP, Anderson CV. Longitudinal study of hearing aid effectiveness. I: Objective measures. J Speech Hear Res. 1993;36(4):808-19. [PMID: 8377493] 
3. Bentler RA, Niebuhr DP, Getta JP, Anderson CV. Longitudinal study of hearing aid effectiveness. II: Subjective measures. J Speech Hear Res. 1993;36(4):820-31. [PMID: 8377494]

4. Cox RM, Alexander GC, Taylor IM, Gray GA. Benefit acclimatization in elderly hearing aid users. J Am Acad Audiol. 1996;7(6):428-41. [PMID: 8972444]

5. Flynn MC, Davis PB, Pogash R. Multiple-channel non-linear power hearing instruments for children with severe hearing impairment: Long-term follow-up. Int J Audiol. 2004;43(8): 479-85. [PMID: 15643742]

6. Humes LE, Halling D, Coughlin M. Reliability and stability of various hearing-aid outcome measures in a group of elderly hearing-aid wearers. J Speech Hear Res. 1996; 39(5):923-35. [PMID: 8898247]

7. Humes LE, Wilson DL. An examination of changes in hearing-aid performance and benefit in the elderly over a 3-year period of hearing-aid use. J Speech Lang Hear Res. 2003;46(1):137-45. [PMID: 12647894]

8. Keidser G, Grant F. Comparing loudness normalization (IHAFF) with speech intelligibility maximization (NALNL1) when implemented in a two-channel device. Ear Hear. 2001;22(6):501-15. [PMID: 11770672]

9. Munro KJ, Lutman ME. Self-reported outcome in new hearing aid users over a 24 -week post-fitting period. Int $\mathrm{J}$ Audiol. 2004;43(10):555-62. [PMID: 15724519]

10. Saunders GH, Cienkowski KM. Acclimatization to hearing aids. Ear Hear. 1997;18(2):129-39. [PMID: 9099562]

11. Surr RK, Cord MT, Walden BE. Long-term versus shortterm hearing aid benefit. J Am Acad Audiol. 1998;9(3): 165-71. [PMID: 9644613] Erratum in: J Am Acad Audiol. 1998 Oct;9(5):398.

12. Turner CW, Bentler RA. Does hearing aid benefit increase over time? J Acoust Soc Am. 1998;104(6):3673-74. [PMID: 9857524]

13. Arlinger S, Billermark E. One year follow-up of users of a digital hearing aid. Brit J Audiol. 1999;33(4):223-32. [PMID: 10509857]

14. Cox RM, Alexander GC. Maturation of hearing aid benefit: Objective and subjective measurements. Ear Hear. 1992; 13(3):131-41. [PMID: 1397752]

15. Gatehouse S. Apparent auditory deprivation effects of late onset: The role of presentation level. J Acoust Soc Am. 1989;86(6):2103-6. [PMID: 2600300]

16. Gatehouse $S$. The time course and magnitude of perceptual acclimatization to frequency responses: Evidence from monaural fitting of hearing aids. J Acoust Soc Am. 1992; 92(3):1258-68. [PMID: 1401514$]$

17. Gatehouse S. Role of perceptual acclimatization in the selection of frequency responses for hearing aids. J Am Acad Audiol. 1993;4(5):296-306. [PMID: 8219296]

18. Horwitz AR, Turner CW. The time course of hearing aid benefit. Ear Hear. 1997;18(1):1-11. [PMID: 9058033]
19. Kuk FK, Potts L, Valente M, Lee L, Picirrillo J. Evidence of acclimatization in persons with severe-to-profound hearing loss. J Am Acad Audiol. 2003;14(2):84-99.

[PMID: 12830844$]$

20. Munro KJ, Lutman ME. The effect of speech presentation level on measurement of auditory acclimatization to amplified speech. J Acoust Soc Am. 2003;114(1):484-95. [PMID: 12880059]

21. Silman S, Silverman CA, Emmer MB, Gelfand SA. Effects of prolonged lack of amplification on speech-recognition performance: Preliminary findings. J Rehabil Res Dev. 1993;30(3):326-32. [PMID: 8126657]

22. Arlinger S, Gatehouse S, Bentler RA, Byrne D, Cox RM, Dirks DD, Humes L, Neuman A, Ponton C, Robinson K, Silman S, Summerfield AQ, Turner CW, Tyler RS, Willot JF. Report of the Eriksholm Workshop on auditory deprivation and acclimatization. Ear Hear. 1996;17(3 Suppl):87S-98. [PMID: 8807279]

23. Palmer CV, Nelson CT, Lindley GA 4th. The functionally and physiologically plastic adult auditory system. J Acoust Soc Am. 1998;103(14):1705-21. [PMID: 9566316]

24. Revoile SG, Holden-Pitt L, Picket JM. Perceptual cues to the voiced-voiceless distinction of final fricatives for listeners with impaired or with normal hearing. J Acoust Soc Am. 1985;77(3):1263-65. [PMID: 3980876]

25. Lindholm JM, Dorman M, Taylor BE, Hannley MT. Stimulus factors influencing the identification of voiced stop consonants by normal-hearing and hearing-impaired adults. J Acoust Soc Am. 1988;83(4):1608-14. [PMID: 3372871$]$

26. Hedrick MS, Schulte L, Jesteadt W. Effect of relative and overall amplitude on perception of voiceless stop consonants by listeners with normal and impaired hearing. J Acoust Soc Am. 1995;98(3):1292-1303. [PMID: 7560503]

27. Hedrick MS, Jesteadt W. Effect of relative amplitude, presentation level, and vowel duration on perception of voiceless stop consonants by normal and hearing-impaired listeners. J Acoust Soc Am. 1996;100(5):3398-3407. [PMID: 8914319$]$

28. Hedrick MS. Effects of acoustic cues on labeling fricatives and affricates. J Speech Lang Hear Res. 1997;40(4):925-38. [PMID: 9263955]

29. Hedrick MS, Carney AE. Effect of relative amplitude and formant transitions on the perception of place of articulation by adult listeners with cochlear implants. J Speech Lang Hear Res. 1997;40(6):1445-57. [PMID: 9430763]

30. Hedrick MS, Ohde RN. Effect of relative amplitude of frication on perception of place of articulation. J Acoust Soc Am. 1993;94(4):2005-26. [PMID: 8227744]

31. Hedrick MS, Rice T. Effect of a single-channel wide dynamic range compression circuit on perception of stop consonant place of articulation. J Speech Lang Hear Res. 2000;43(5):1174-84. [PMID: 11063239] 
32. Hedrick MS, Younger MS. Perceptual weighting of relative amplitude and formant transition cues in aided CV syllables. J Speech Lang Hear Res. 2001;44(5):964-74. [PMID: 11708536]

33. Hedrick MS, Younger MS. Labeling of /s/ and /sh/ by listeners with normal and impaired hearing, revisited. J Speech Lang Hear Res. 2003;46(3):636-48. [PMID: 14696991]

34. Yund EW, Buckles KM. Multichannel compression hearing aids: Effect of number of channels on speech discrimination in noise. J Acoust Soc Am. 1995;97(2):1206-23. [PMID: 7876443]

35. Yund EW, Buckles KM. Enhanced speech perception at low signal-to-noise ratios with multichannel compression hearing aids. J Acoust Soc Am. 1995;97(2):1224-40. [PMID: 7876444]

36. Yund EW, Buckles KM. Discrimination of multichannelcompressed speech in noise: Long-term learning in hearing-impaired subjects. Ear Hear. 1995;16(4):417-27. [PMID: 8549897]

37. American National Standard Specification for Audiometers, American National Standards Institute, Inc. New York: Acoustical Society of America; 1996.

38. Folstein MF, Folstein SE, McHugh PR. "Mini-Mental State." A practical method for grading the cognitive status of patients for the clinician. J Psychiatr Res. 1975;12(3): 189-98. [PMID: 1202204$]$

39. Barfod J. Multichannel compression hearing aids: Experiments and consideration on clinical applicability. Scand Audiol Suppl. 1978;(6):315-40. [PMID: 292146]

40. Byrne D, Dillon H. The National Acoustic Laboratories' (NAL) new procedure for selecting the gain and frequency response of a hearing aid. Ear Hear. 1986;7(4):257-65. [PMID: 3743918]

41. Resnick SB, Dubno JR, Hoffnung S, Levitt H. Phoneme errors on a nonsense syllable test. J Acoust Soc Am. 1975;58(Suppl 1):114.

42. Dubno JR, Dirks DD. Evaluation of hearing impaired listeners using a nonsense syllable test 1 . Test reliability. J Speech Hear Res. 1982;25(1):135-41. [PMID: 7087416]

43. Dubno JR, Dirks DD, Langhover LR. Evaluation of hearingimpaired listeners using a nonsense syllable test. II. Syllable recognition and consonant confusion patterns. J Speech Hear Res. 1982;25(1):141-48. [PMID: 7087417]

44. Walker G, Byrne D, Dillon H. Learning effects with a closed set nonsense syllable test. Austral J Audiol. 1982; $4: 27-31$.
45. Walker G, Byrne D, Dillon H. The effects of multichannel compression/expansion amplification on the intelligibility of nonsense syllables in noise. J Acoust Soc Am. 1984;76(3): 746-57. [PMID: 6491047]

46. Cox RM, Gilmore C, Alexander GC. Comparison of two questionnaires for patient-assessed hearing aid benefit. J Am Acad Audiol. 1991;2(3):134-45. [PMID: 1768881]

47. Walden BE, Demorest ME, Hepler EL. Self-report approach to assessing benefit derived from amplification. J Speech Hear Res. 1984;27(1):49-56. [PMID: 6717006]

48. Dubno JR, Levitt H. Predicting consonant confusions from acoustic analysis. J Acoust Soc Am. 1981;69(1):249-61. [PMID: 7217523$]$

49. Dubno JR, Ahlstrom JB. Masked thresholds and consonant recognition in low-pass maskers for hearing-impaired and normal-hearing listeners. J Acoust Soc Am. 1995;97(4): 2430-41. [PMID: 7714260]

50. Wang MD, Bilger RC. Consonant confusions in noise: A study of perceptual features. J Acoust Soc Am. 1973; 54(15):1248-66. [PMID: 4765809]

51. Edwards B. The distortion of auditory perception by sensorineural hearing impairment. Audiol Online. 2003; Mar 10:1-5.

52. Yund EW, Simon HJ, Efron R. Speech discrimination with an 8-channel compression hearing aid and conventional aids in background of speech-band noise. J Rehabil Res Dev. 1987;24(4):160-80. [PMID: 3430375]

53. Stecker GC, Bowman GA, Yund EW, Herron TJ, Roup CM, Woods DL. Perceptual training improves syllable identification in new and experienced hearing aid users. J Rehabil Res Dev. 2006;43(4):537-52.

54. Gilbert CD, Sigman M, Crist RE. The neural basis of perceptual learning. Neuron. 2001;31(5):681-97. [PMID: 11567610]

55. Goldstone RL. Perceptual learning. Annu Rev Psychol. 1998;49:585-612. [PMID: 9496632]

56. Cox RM. On the evaluation of a new generation of hearing aids. J Rehabil Res Dev. 1993;30(3):297-304. [PMID: 8126654$]$

57. Van Tasell DJ. Hearing loss, speech, and hearing aids. J Speech Hear Res. 1993;36(2):228-44. [PMID: 8487516]

Submitted for publication June 14, 2005. Accepted in revised form January 13, 2006. 